\title{
Produtividade de tambaqui criado em tanque-rede com diferentes taxas de alimentação
}

\author{
Tambaqui productivity reared in cages with different feeding rations
}

\author{
Edsandra Campos Chagas ${ }^{\mathrm{I}}$ Levy de Carvalho Gomes ${ }^{\mathrm{I}}$ \\ Heitor Martins Júnior ${ }^{\text {II }}$ Rodrigo Roubach ${ }^{\mathrm{II}, \mathrm{III}}$
}

RESUMO

O objetivo deste trabalho foi avaliar o efeito da taxa de alimentação sobre a produtividade e os níveis de glicose sangüínea, proteínas totais e triglicérides plasmáticos de juvenis de tambaqui (Colossoma macropomum) criados em tanquerede. Juvenis de tambaqui foram criados durante 150 dias recebendo alimentação nas taxas de 1, 3 e 5\% do peso vivo dia $^{-1}$ dividida em duas refeições, seis dias por semana. As taxas de alimentação não apresentaram efeito sobre o fator de condição e sobrevivência. O ganho de peso e produção dos peixes que receberam as taxas de alimentação de 3 e 5\% foram significativamente maiores que os do que receberam a taxa de $1 \%$ do peso vivo/dia. Entretanto, a conversão alimentar foi significativamente superior na taxa de 1\%, e os valores de conversão alimentar obtidos nas taxas de 3 e 5\% do peso vivo dia ${ }^{-1}$, por si, tornam a criação economicamente inviável. Não ocorreu alteração significativa nos níveis de glicose sangüínea, triglicérides e proteínas plasmáticas de tambaquis submetidos aos diferentes tratamentos. A taxa de alimentação adequada para a produtividade de juvenis de tambaqui criados em tanques-rede instalados em lagos de várzea, pesando inicialmente $200 \mathrm{~g}$, é de $1 \%$ do peso vivo dia ${ }^{-1}$.

Palavras-chave: Colossoma macropomum, crescimento, nutrição, piscicultura.

\section{ABSTRACT}

This research was aimed at evaluating the effect of feeding rates on productivity and blood glucose levels, triglycerides and total plasma proteins of tambaqui (Colossoma macropomum) reared in cages. Tambaqui juveniles were reared during 150 days fed at 1, 3 and 5\% of body weight per day allocated with two meals, six days a week. The feeding rates did not show any effects on the condition factor and survival. Weight gain and production of fish that were fed at 3 and 5\% were significantly higher than the fish that received $1 \%$ of body weight per day. Although, feed conversion was significantly higher at the ratio of $1 \%$ of body weight per day, and the values of feed conversion obtained at the ratios of 3 and 5\% per se make the culture of tambaqui unprofitable. No alterations were observed in the levels of blood glucose, triglycerides and total plasma protein of tambaqui from the different treatments. The feeding-ratio productivity adequate for tambaqui juveniles, when reared in cages in varzea lakes with an initial weigh of $200 \mathrm{~g}$, is $1 \%$ of body weight per day.

Key words: Colossoma macropomum, growth, nutrition, fish culture.

\section{INTRODUÇÃO}

O tambaqui, Colossoma macropomum, é uma espécie nativa da Amazônia que apresenta bom desempenho em criação intensiva, sendo a mais cultivada na Região Norte do Brasil (VAL et al., 2000). Criações de tambaqui em tanques-rede instalados em lagos de várzea da Amazônia Central têm alcançado alta produtividade (CHAGAS et al., 2003), sendo apontadas como uma atividade promissora para a sua criação entre as populações ribeirinhas da Amazônia.

O manejo alimentar é de grande importância para êxito na criação de peixes. A adoção de estratégias

\footnotetext{
'Embrapa Amazônia Ocidental, CP 319, 69011-970, Manaus, AM, Brasil. E-mail: edsandra@cpaa.embrapa.br. Autor para correspondência.

"Instituto Nacional de Pesquisas da Amazônia (INPA), Coordenação de Pesquisas em Aquacultura (CPAQ), CP 478, 69083-000, Manaus, AM, Brasil.

IIISecretaria Especial de Aqüicultura e Pesca (SEAP/PR), Diretoria de Desenvolvimento da Aqüicultura/DIDAQ, Esplanada dos Ministérios Bloco D, sala 239, 70043-900, Brasília, DF, Brasil.
} 
de alimentação adequadas, nas diferentes fases de vida dos peixes, permite melhorar o seu crescimento, a sua sobrevivência e conversão alimentar, contribuindo para reduzir o desperdício de ração, o que prejudica a qualidade da água de cultivo e a produção (GODDARD, 1996; CHO et al., 2003).

Para estabelecer estratégias eficientes de manejo alimentar, deve-se avaliar a taxa e freqüência de alimentação, além de métodos adequados de dispersão do alimento (GODDARD, 1996). A taxa de alimentação influencia tanto o crescimento quanto a eficiência alimentar de peixes cultivados, e o seu crescimento é diretamente proporcional à taxa de arraçoamento empregada (NG et al., 2000; MIHELAKAKIS et al., 2002; EROLDOGAN et al., 2004).

Espécies como o pacu (Piaractus mesopotamicus), "European sea bass" (Dicentrarchus labrax L.) e "Gilthead sea bream” (Sparus aurata) (BORGHETTI \& CANZI, 1993; MIHELAKAKIS et al., 2002; EROLDOGAN et al., 2004) têm suas estratégias de alimentação definidas para uma melhor utilização do alimento e controle da taxa de crescimento. Foram determinadas as exigências nutricionais do tambaqui quanto aos macro e micronutrientes em diferentes fases de vida (MACEDO-VIEGAS et al., 1996; CHAGAS \& VAL, 2003); contudo, existem poucas informações sobre sua taxa de alimentação e freqüência de arraçoamento em criação intensiva.

O objetivo deste trabalho foi avaliar o efeito da taxa de alimentação sobre a produtividade e os níveis de glicose sangüínea, proteínas totais e triglicérides plasmáticos de juvenis de tambaqui (Colossoma macropomum) criados em tanque-rede.

\section{MATERIAL E MÉTODOS}

Esse estudo foi conduzido em nove tanquesrede de $6 \mathrm{~m}^{3}$ (2,0 x 2,0 x 1,5m), com tamanho de malha de $20 \mathrm{~mm}$ e sistema de flutuação constituído por toras de madeira de baixa densidade. Os tanques-rede foram instalados no lago Ariauzinho (Iranduba - AM) ( $3^{\circ} 14^{\prime}$ 16.40" de latitude sul e $60^{\circ} 14^{\prime} 18.20 "$ de longitude oeste), um típico lago de várzea da Amazônia, de formato dendrítico, com flutuações do nível da água de até $8 \mathrm{~m}$ entre a seca e a cheia do rio Solimões. Juvenis de tambaqui $(197 \pm 48 \mathrm{~g}$ e $18 \pm 1 \mathrm{~cm})$ foram distribuídos segundo um delineamento inteiramente casualizado em três tratamentos, cada um com três repetições referentes às taxas de alimentação de 1,3 e $5 \%$ do peso vivo dia $^{-1}$, numa densidade de 15 peixes $\mathrm{m}^{-3}$ (90 peixes por tanque). A alimentação foi fornecida em duas refeições diárias (8 e 16h), seis dias por semana, utilizando-se ração comercial extrusada para peixes onívoros, com $28 \%$ de proteína bruta. A temperatura da água e o oxigênio dissolvido foram monitorados três vezes por semana por meio de um monitor YSI 55. A alcalinidade foi determinada pelo método titrimétrico do EDTA e a dureza por titrimetria com alaranjado de metila (APHA, 1992), o pH eletrometricamente e a amônia total pelo método de endofenol (APHA, 1992). Essas avaliações foram feitas quinzenalmente.

Biometrias mensais, utilizando 15\% dos peixes de cada tanque-rede, foram realizadas para ajustar a quantidade de ração a ser fornecida e para obtenção dos parâmetros de crescimento em peso (g) e comprimento padrão (cm), ganho de peso (peso final - peso inicial), conversão alimentar aparente (alimento consumido, g ganho de peso $^{-1}$, g), fator de condição (peso comprimento-3) e produção $\left(\mathrm{kg} \mathrm{m}^{-3}\right)$. A sobrevivência dos peixes foi avaliada após 150 dias de criação. O custo de alimentação foi obtido pela razão valor da ração/kg de peixe produzido. Para cálculo de custos de alimentação, utilizou-se o preço de venda da ração no mercado de Manaus em abril/2004 (U\$ 0,40 $\left.\mathrm{kg}^{-1}\right)$.

No início e final do período de criação, três peixes de cada repetição (nove peixes de cada tratamento) foram amostrados para determinação dos parâmetros fisiológicos. Para isso, os peixes foram anestesiados com benzocaína, 100 $\mathrm{mg} \mathrm{L}^{-1}$, e o sangue coletado por meio de punção no vaso caudal com seringas heparinizadas, sendo as amostras acondicionadas em gelo fundente, até o processamento. A glicose sangüínea $\left(\mathrm{mg} \mathrm{dL}^{-1}\right)$ foi determinada com glicosímetro digital (Advantage II, Roche $^{\circledR}$ ). Em seguida, o sangue foi centrifugado a 823g por cinco minutos. No plasma sobrenadante, foram determinadas as concentrações de triglicérides (mg $\mathrm{dL}^{-1}$ ) e proteínas totais $\left(\mathrm{g} \mathrm{dL}^{-1}\right)$, utilizando-se kit comercial específico (Doles $^{\circledR}$, Goiás, Brasil).

Os resultados obtidos estão expressos como média \pm desvio padrão. A significância das diferenças obtidas entre as médias para os diferentes tratamentos foi estabelecida por análise de variância de um fator. As médias foram comparadas pelo teste de Tukey com intervalo de confiança de 5\%, utilizando o programa Sigma Stat 2.0.

\section{RESULTADOS E DISCUSSÃO}

Os valores de temperatura e oxigênio dissolvido não apresentaram diferença significativa entre os tratamentos, estando próximos aos valores recomendados para a criação de tambaquis (IZEL \& MELO, 2004). Desta forma, os dados foram agrupados para representar o perfil de oxigênio e a temperatura do lago (Figura 1). A temperatura variou entre 28,4 e 33,13을 


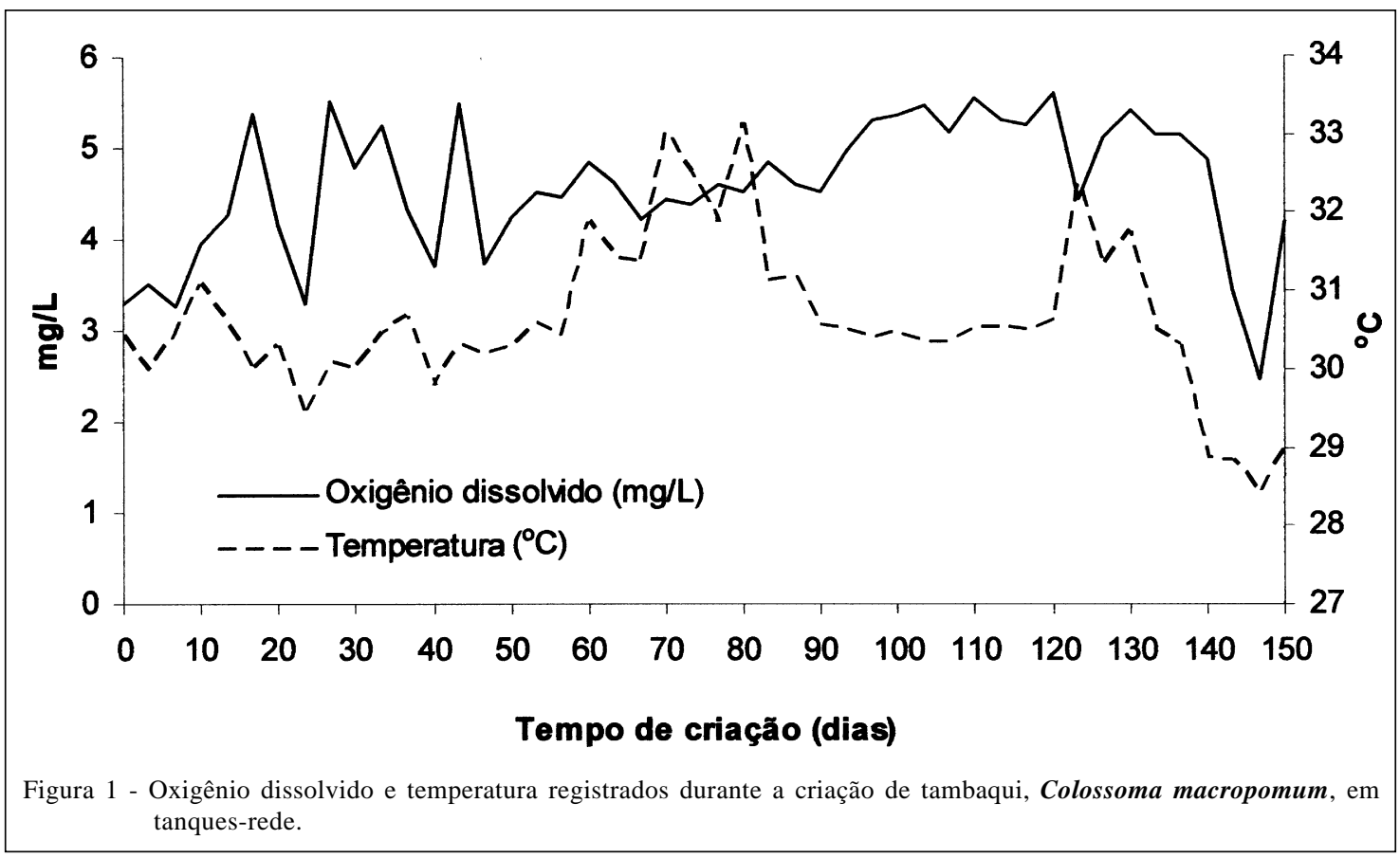

e o oxigênio dissolvido entre 2,59 e 5,72mg L $\mathrm{m}^{-1}$ (Figura 1). A amônia e o pH se mantiveram estáveis durante todo o período de criação (Figura 2). A alcalinidade e a dureza apresentaram um padrão similar. Os valores destas variáveis foram estáveis durante o estudo e no último mês aumentaram, reflexo da entrada de água do rio Solimões no lago.

A qualidade da água do lago Ariauzinho é muito semelhante à de outros lagos de várzea da região
(MELACK \& FISHER, 1983), lagos que são o habitat natural da espécie; dessa forma, o tambaqui está adaptado a estas características. Ambientes naturais devem ser criteriosamente selecionados para instalação de tanque-rede, uma vez que é impossível manejar a qualidade da água dos mesmos (BEVERIDGE, 1996; HUGUENIN, 1997). A principal variável a ser avaliada é o oxigênio dissolvido, que, neste ambiente, pode atingir valores indesejáveis, como observado no final do

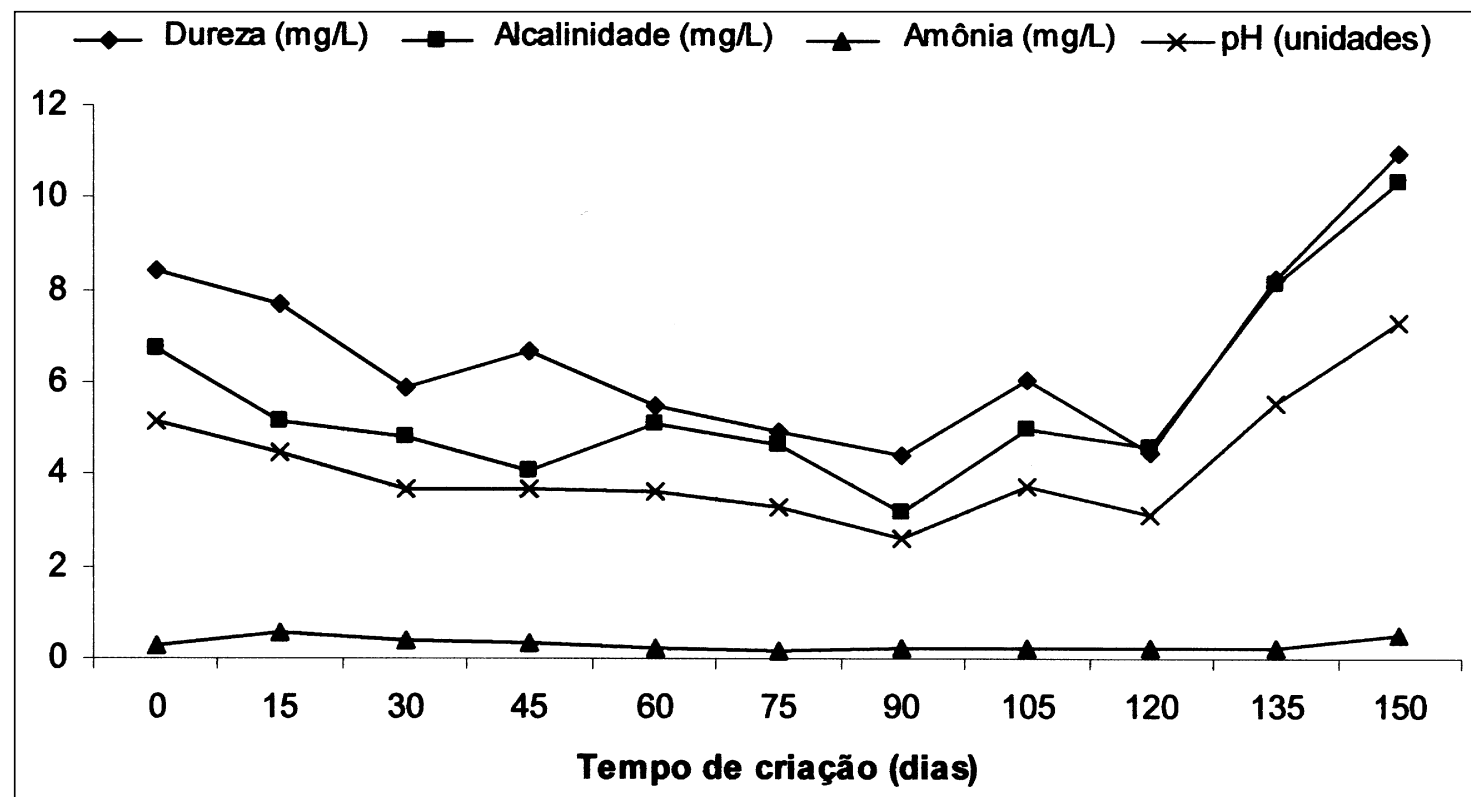

Figura 2 - pH, alcalinidade, dureza e amônia registrados durante a criação de tambaqui, Colossoma macropomum, em tanques-rede.

Ciência Rural, v.37, n.4, jul-ago, 2007. 
período de estudo. A alcalinidade, a dureza, o pH e a amônia estiveram dentro da faixa ótima para criação do tambaqui. Esta deve ser uma constante para criação em tanque-rede neste tipo de ambiente na Amazônia.

A sobrevivência dos juvenis de tambaqui em todas as taxas de alimentação testadas foi superior a 97\% (Tabela 1); resultado este similar ao observado para a espécie gilthead sea bream, que obteve uma taxa de sobrevivência ao redor de 95\% (MIHELAKAKIS et al., 2002).

O aumento de peso e comprimento dos juvenis de tambaqui foi maior nas taxas de alimentação de 3 e $5 \%$ (Figura 3A e B). Nos primeiros trinta dias de criação, foi possível observar diferença significativa no peso dos peixes, e após 150 dias de criação, os peixes que receberam as taxas de alimentação de 3 e $5 \%$ do peso vivo dia $^{-1}$ apresentaram pesos médios de $687,27 \pm 32,80$ e $791,48 \pm 39,08 \mathrm{~g}$, respectivamente, significativamente maiores que aquele registrado para os peixes recebendo a taxa de $1 \%$ do peso vivo dia $^{-1}$

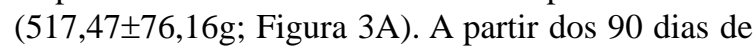
criação, os peixes que receberam as taxas de alimentação de 3 e $5 \%$ foram mais compridos $(26,66 \pm 0,40$ e $27,88 \pm 0,46 \mathrm{~cm}$, respectivamente) que os que receberam ração na taxa de $1 \%$ do peso vivo dia $^{-1}(24,54 \pm 1,17 \mathrm{~cm}$, Figura 3B).

O desempenho do tambaqui obtido neste estudo para as três taxas de alimentação foi satisfatório. Em 120 dias de criação na densidade de 15 peixes $\mathrm{m}^{-3}$, os peixes das taxas de alimentação 3 e $5 \%$ apresentaram peso médio superior (604 e 677g, respectivamente) ao relatado por CHAGAS et al. (2003) no mesmo lago de várzea, na densidade de 25 peixes $/ \mathrm{m}^{3}$. Nesse relato, os tambaquis alcançaram um peso médio final de $540 \mathrm{~g}$ quando alimentados até a saciedade aparente por 120 dias, ficando próximo ao obtido neste estudo para a menor taxa de alimentação avaliada (483g; Figura 3A). $\mathrm{O}$ ganho de peso e produção de juvenis de tambaqui foram afetados pela taxa de alimentação empregada (Tabela 1). Os melhores valores de ganho de peso (490,3 e $594,5 \mathrm{~g})$ e produção $\left(10,15\right.$ e $\left.11,83 \mathrm{~kg} \mathrm{~m}^{-3}\right)$ foram alcançados pelos peixes que receberam as taxas de $3 \mathrm{e}$ $5 \%$ do peso vivo/dia (Tabela 1), valores esses significativamente diferentes que os dos peixes que receberam a taxa de alimentação de $1 \%$ do peso vivo dia $^{-1}$.

Estudos conduzidos com tambaquis em tanques-rede na densidade de 34 peixes $\mathrm{m}^{-3}$ obtiveram uma produção de 14,4kg m-3 (CHELLAPA et al., 1995). Já pacus alimentados com a taxa de alimentação de 5\% apresentaram maior eficiência produtiva, com a temperatura na faixa de 27 a $30^{\circ} \mathrm{C}$, quando comparados a animais com taxas de 1 e $3 \%$ (BORGHETTI \& CANZI, 1993).

O crescimento não é a única variável que deve ser considerada na determinação da taxa de alimentação ótima. A eficiência na conversão do alimento também é um bom indicador, sendo relatado que a porcentagem de eficiência na conversão do alimento diminui com o aumento na taxa de alimentação (NG et al., 2000; EROLDOGAN et al., 2004). A conversão alimentar tem consequiência direta nos custos de produção, pois a ração representa cerca de $30-60 \%$ dos custos operacionais da criação de peixes em tanquerede (HUGUENIN, 1997).

Os peixes que receberam a taxa de $1 \%$ apresentaram uma maior eficiência na conversão alimentar, quando comparados aos que receberam $3 \mathrm{e}$ $5 \%$ do peso vivo dia $^{-1}$ (Tabela 1). Segundo ZOCCARATO et al. (1994) e VAN HAM et al. (2003), os peixes tendem a otimizar a sua digestão para extrair o alimento de forma mais eficiente quando alimentados com taxas de alimentação baixas, o que está de acordo com os resultados obtidos, pois o fornecimento de ração para tambaquis em taxas superiores a $1 \%$ do peso vivo $\operatorname{dia}^{-1}$, nas condições de lago de várzea, causa

Tabela 1 - Desempenho de tambaqui, Colossoma macropomum, alimentado em tanques-rede com três diferentes taxas de alimentação durante 150 dias.

\begin{tabular}{lccc}
\hline & \multicolumn{3}{c}{ Taxas de alimentação (\% peso vivo/dia) } \\
\cline { 2 - 4 } Variáveis & 1 & 3 & 5 \\
\hline Ganho de peso (g) & $320,5 \pm 76,2 \mathrm{a}$ & $490,3 \pm 32,8 \mathrm{~b}$ & $594,5 \pm 39,1 \mathrm{~b}$ \\
Conversão alimentar aparente & $1,98 \pm 0,22 \mathrm{a}$ & $4,86 \pm 0,06 \mathrm{~b}$ & $7,07 \pm 0,05 \mathrm{c}$ \\
Fator de condição & $3,41 \pm 0,03 \mathrm{a}$ & $3,57 \pm 0,02 \mathrm{a}$ & $3,84 \pm 0,4 \mathrm{a}$ \\
Sobrevivência (\%) & $98,52 \pm 1,70 \mathrm{a}$ & $97,78 \pm 2,94 \mathrm{a}$ & $99,26 \pm 0,64 \mathrm{a}$ \\
Produção $\left(\mathrm{kg} / \mathrm{m}^{3}\right)$ & $7,72 \pm 0,97 \mathrm{a}$ & $10,15 \pm 0,45 \mathrm{~b}$ & $11,83 \pm 0,74 \mathrm{~b}$ \\
Custo de alimentação (U\$/kg peixe)* & $0,79 \pm 0,08 \mathrm{a}$ & $1,95 \pm 0,02 \mathrm{~b}$ & $2,83 \pm 0,20 \mathrm{c}$ \\
\hline
\end{tabular}

Em cada linha, médias seguidas da mesma letra não diferem entre si pelo teste de Tukey a 5\% de probabilidade.

$* \mathrm{U} \$ 1$ = R\$ 2,12.

Ciência Rural, v.37, n.4, jul-ago, 2007. 


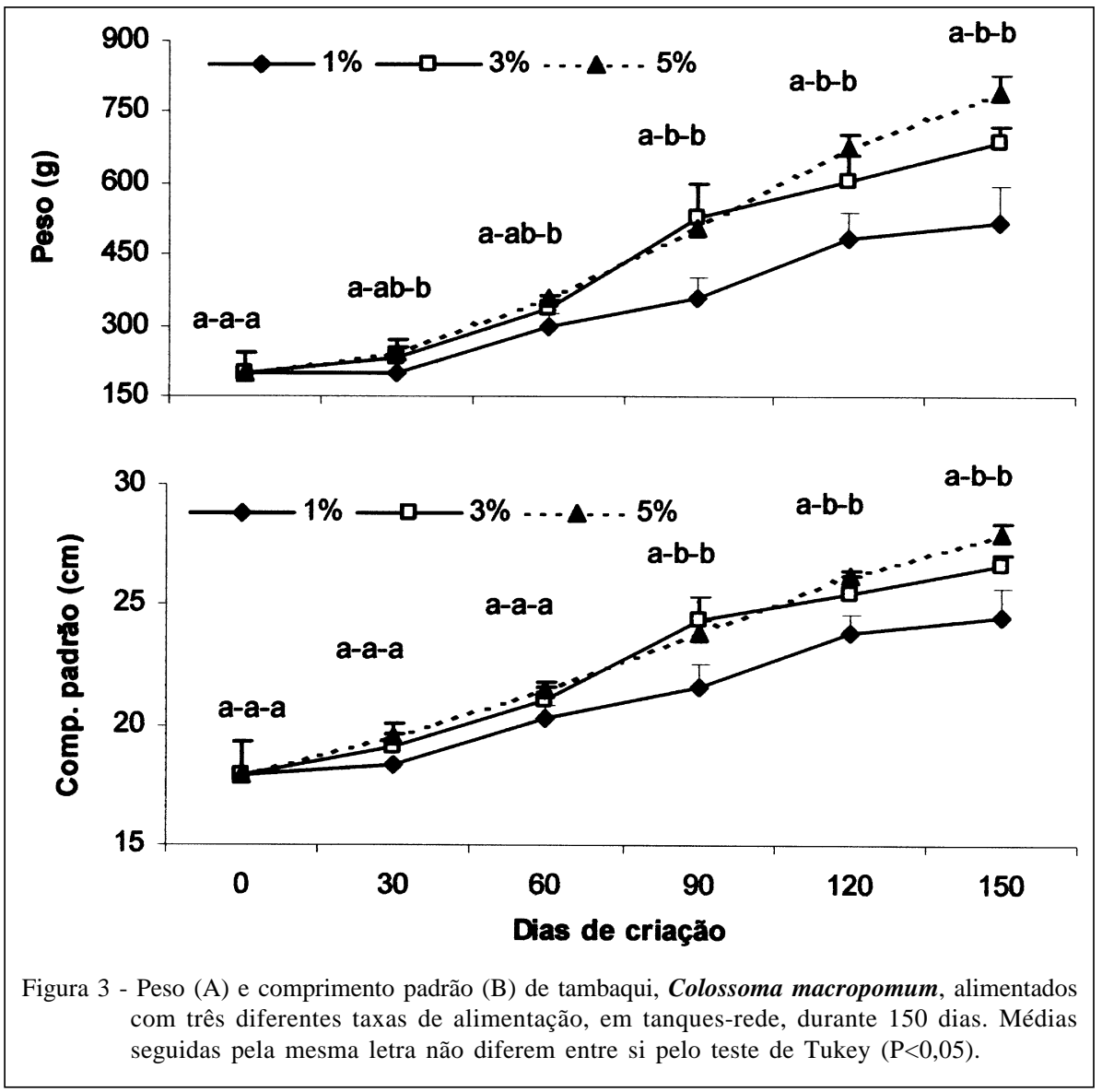

desperdício de ração, sendo essas sobras visivelmente maiores nos dias seguintes ao ajuste na alimentação. Maior eficiência na conversão do alimento foi obtido para "European sea bass", com a taxa de 3,5\% do peso vivo/dia (EROLDOGAN et al., 2004), e para “yellowtail flounder" (Limanda ferruginea), com o fornecimento de ração na taxa de $1 \%$ do peso vivo dia $^{-1}$; valor abaixo da taxa utilizada por esta espécie para máximo crescimento (3\%) (PUVANENDRAN et al., 2003). Nas condições deste estudo, embora a taxa de $1 \%$ do peso vivo/dia apresente a melhor conversão alimentar para tambaquis criados em tanque-rede, o ganho de peso e a produção ficaram abaixo dos obtidos na taxa de 3\%, que seria a taxa adequada para alcançar o crescimento ótimo em viveiros (MELO et al., 2001). Contudo, apesar de as taxas de 3 e $5 \%$ do peso vivo dia $^{-1}$ apresentarem valores superiores de ganho de peso e produção, elas se tornam impraticáveis pela conversão alimentar obtida, necessitando de uma ingestão de 4,86 e 7,07kg de ração para alcançar $1 \mathrm{~kg}$ de peso corporal. Isso implica o aumento dos custos com ração para cerca de 2,4 a 3,5 maior em relação ao custo da taxa de $1 \%$ do peso vivo $\mathrm{dia}^{-1}$, sendo esses resultados significativamente diferentes (Tabela 1). Este resultado mostra que a criação de tambaqui nas taxas de alimentação de 3 e $5 \%$ do peso vivo dia ${ }^{-1}$ é economicamente inviável por despender U\$ 1,95 e U\$ 2,83 em ração, na produção de $1 \mathrm{~kg}$ de peixe, que no mercado de Manaus é vendido por $\mathrm{U} \$ 2,36 \mathrm{~kg}^{-1}$. O custo com alimentação para os peixes produzidos com taxa de alimentação de $1 \%$ do peso vivo/dia foi de U\$ 0,79, sendo estes peixes vendidos no mercado de Manaus por U\$ 1,89 $\mathrm{kg}^{-1}$. O custo com ração representou $59 \%$ do custo total de produção do tambaqui criado em tanque-rede, no mesmo lago, na densidade de 20 peixes $\mathrm{m}^{-3}$ (GOMES et al., 2006). Desta forma, utilizou-se esse trabalho como referência para calcular o custo total de produção e obteve-se que apenas os peixes criados com $1 \%$ do peso vivo dia ${ }^{-1}$ apresentam custo de total de produção (U\$ 1,34) inferior ao preço de venda.

O fator de condição permite avaliar o grau de atividade alimentar de uma espécie, verificando se ela está ou não fazendo bom uso da fonte nutricional, sendo, portanto, um bom indicador de sua condição fisiológica (NG et al., 2000; MIHELAKAKIS et al., 2002). Neste estudo, o fator de condição não apresentou 
diferença significativa entre as três taxas de alimentação avaliadas para juvenis de tambaqui (Tabela 1). Quando os peixes recebem alimentação abaixo das suas necessidades nutricionais ou ficam longos períodos sem receber alimentos, a manutenção dos processos vitais se dá à custa da mobilização das reservas energéticas (JOBLING, 1994). O fornecimento de alimento nas três taxas de alimentação atendeu s exigências mínimas do tambaqui, não necessitando desta forma mobilizar as suas reservas energéticas, pois todos os parâmetros fisiológicos avaliados (glicose, triglicérides e proteínas) não apresentaram diferença significativa entre as diferentes taxas de alimentação avaliadas. Os valores médios destes parâmetros foram: glicose $\left(60,09 \pm 2,20 \mathrm{mg} \mathrm{dL}^{-1}\right)$, triglicérides (216,15 $\left.\pm 76,23 \mathrm{mg} \mathrm{dL}^{-1}\right)$ e proteínas $\left(2,45 \pm 0,44 \mathrm{~g} \mathrm{dL} \mathrm{d}^{-1}\right)$.

A mobilização de proteínas também é uma estratégia para manutenção dos processos vitais em peixes quando submetidos a períodos de restrição alimentar (SHERIDAN \& MOMMSEN, 1991). Para tambaquis, não foi observada nenhuma alteração nos níveis de proteínas, mesmo nos peixes que receberam a menor taxa de alimentação. As concentrações de triglicérides plasmáticos não apresentaram diferenças significativas entre os peixes alimentados nas diferentes taxas de alimentação. Valores de triglicérides próximos aos observados para o tambaqui foram registrados para pacus criados sob diferentes regimes de alimentação (SOUZA et al., 2000). Em percas (Perca fluviatilis), os valores de triglicérides apresentaram uma correlação positiva com a taxa de alimentação, Ò fotoperíodo e A temperatura da água (KAVADIAS et al., 2003). Aumentos nos níveis de glicose em peixes estão relacionados a uma série de estressores como variações de temperatura, transporte e manuseio (ACERETE et al., 2004; LERMEN et al., 2004; URBINATI, et al. 2004). A mobilização da glicose tem por objetivo fornecer energia extra para o animal resistir durante o período de distúrbio. Tambaquis não apresentaram sinais de estresse nas diferentes taxas de alimentação avaliadas, e os valores de glicose obtidos são semelhantes aos observados na criação da espécie em diferentes densidades de estocagem no sistema de tanque-rede (CHAGAS et al., 2003).

Os peixes têm pouca necessidade de mobilizar energia endógena quando mantidos em condições normais de cultivo, com fornecimento adequado de ração (COOK et al., 2000). Essas informações corroboram os resultados deste estudo, pois o fornecimento de alimento nas três taxas de alimentação (1, 3 e 5\% do peso vivo dia $^{-1}$ ) foi suficiente para atender às exigências do tambaqui, não sendo necessário, portanto, a mobilização das reservas energéticas, permitindo desta forma alcançar um desempenho satisfatório do tambaqui em sistema de tanque-rede.

\section{CONCLUSÃO}

A taxa de alimentação adequada para a produtividade de juvenis de tambaqui criados por 150 dias em tanques-rede instalados em lagos de várzea, pesando inicialmente $200 \mathrm{~g}$ e alimentados duas vezes por dia, é a de $1 \%$ do peso vivo dia $^{-1}$.

\section{AGRADECIMENTOS}

Aos Srs. José Pereira, Mário Kokay e Márcia Pessoa, da Embrapa Amazônia Ocidental, e aos bolsistas do Laboratório de Piscicultura, pelo auxílio na condução dos experimentos. Ao apoio financeiro do projeto TANRE FINEP/ FUCAPI e BASA. Ao CNPq, pela bolsa de produtividade em pesquisa concedida ao último autor.

\section{REFERÊNCIAS}

ACERETE, L. et al. Physiological responses in Eurasian perch (Perca fluviatilis, L.) subjected to stress by transport and handling. Aquaculture, v.237, p.167-178, 2004.

APHA (American Public Health Association, American Water Works Association, Water environment Federation). Standard methods for the examination of water and wastewater. New York: American Public Health Association, 1992. 1050p.

BEVERIDGE, M.C.M. Cage aquaculture. Oxford: Fishing News Books, 1996. 346p.

BORGHETTI, J.R.; CANZI, C. The effect of water temperature and feeding rate on the growth rate of pacu (Piaractus mesopotamicus) raised in cages. Aquaculture, v.114, p.93101, 1993.

COOK, J.T. et al. Effect of food deprivation on oxygen consumption and body composition of growth-enhanced transgenic Atlantic salmon (Salmo salar). Aquaculture, v.188, p.47-63, 2000.

CHAGAS, E.C. et al. Desempenho e estado de saúde de tambaquis cultivados em tanques-rede sob diferentes densidades de estocagem. In: URBINATI, E.C.; CYRINO, J.E.P. SIMPÓSIO BRASILEIRO DE AQÜICULTURA, 12., 2003, Jaboticabal, SP. Anais... Jaboticabal: AQUABIO, 2003. Cap.9, p.83-93.

CHAGAS, E.C.; VAL, A.L. Efeito da vitamina C no ganho de peso e em parâmetros hematológicos de tambaqui. Pesquisa Agropecuária Brasileira, v.38, p.397-402, 2003.

CHELLAPA, S. et al. Growth and production of the Amazonian tambaqui in fixed cages under different feeding regimes. Aquaculture International, v.3, p.11-21, 1995.

CHO, S.H. et al. Effects of feeding rate and feeding frequency on survival, growth, and body composition of Ayu post-larvae Plecoglossus altivelis. Journal of the World Aquaculture Society, v.34, p.85-91, 2003.

EROLDOGAN, O.T. et al. Optimum feeding rates for European sea bass Dicentrarchus labrax L. reared in seawater and freshwater. Aquaculture, v.231, p.501-515, 2004. 
GODDARD, S. Feed management in intensive aquaculture. New York: Chapman \& Hall, 1996. 194p.

GOMES, L.C. et al. Cage culture of tambaqui (Colossoma macropomum) in a central Amazon floodplain lake. Aquaculture, v.253, p.374-384, 2006.

HUGUENIN, J. The design, operations and economics of cage culture systems. Aquacultural Engineering, v.16, p.167203, 1997.

IZEL, A.C.U.; MELO, L.A.S. Criação de tambaqui (Colossoma macropomum) em tanques escavados no estado do Amazonas. Manaus: Embrapa Amazônia Ocidental, 2004. 19p. (Embrapa Amazônia Ocidental. Documentos, 32).

JOBLING, M. Fish bioenergetics. London: Chapman \& Hall, 1994. 309p.

KAVADIAS, S. et al. Annual cycles of growth rate, feeding rate, food conversion, plasma glucose and plasma lipids in a population of European sea bass (Dicentrarchus labrax L.) farmed in floating marine cages. Journal of Applied Ichthyology, v.19, p.29-34, 2003.

LERMEN, C.L. et al. Effect of different temperature regimes on metabolic and blood parameters of silver catfish Rhamdia quelen. Aquaculture, v.239, p.497-507, 2004

MACEDO-VIEGAS, E.M. et al. Níveis de proteína bruta em dietas para o crescimento do tambaqui Colossoma macropomum, Cuvier, 1818 (Pisces Characidae). Revista Unimar, v.18, p.321-333, 1996.

MELACK, J.M.; FISHER, T.R. Diel oxygen variations and their ecological implications in an Amazon floodplain lakes. Archiv fur Hydrobiologie, v.98, p.422-442, 1983.

MELO, L.A.S. et al. Criação de tambaqui (Colossoma macropomum) em viveiros de argila/barragens no Estado do Amazonas. Embrapa Amazônia Ocidental, 2001. 30p. (Embrapa Amazônia Ocidental. Documentos, 18).
MIHELAKAKIS, A. et al. Optimization of feeding rate of hatchery-produced juvenile gilthead sea bream Sparus aurata. Journal of the World Aquaculture Society, v.33, p.169175, 2002

NG, W.K. et al. Effects of feeding rate on growth, feed utilization and body composition of a tropical bagrid catfish. Aquaculture International, v.8, p.19-29, 2000

PUVANENDRAN, V. et al. Food ration requirements of $\mathrm{O}^{+}$ yellowtail flounder Limanda ferruginea (Storer) juveniles. Aquaculture, v.220, p.459-475, 2003.

SHERIDAN, M.A.; MOMMSEN, T.P. Effects of nutritional state on in vivo lipid and carbohydrate metabolism of coho salmon, Oncorhynchus kisutch. General and Comparative Endocrinology, v.81, p.473-483, 1991.

SOUZA, V.L. et al. Effect of food restriction and refeeding on energy stores and growth of Pacu, Piaractus mesopotamicus (Characidae). Journal of Aquaculture in the Tropics, v.15, p.371-379, 2000.

URBINATI, E.C. et al. Loading and transport stress in juvenile matrinxã (Brycon cephalus) at various densities. Aquaculture, v.229, p.389-400, 2004.

VAL, A.L. et al. Situação atual da aqüicultura na região Norte. In: VALENTI, W.C. et al. Aqüicultura no Brasil. Brasília: CNPq, 2000. Cap.7, p.247-266.

VAN HAM, E.H. et al. The influence of temperature and ration on growth, feed conversion, body composition and nutrition retention of juvenile turbot (Scohthalmus maximus). Aquaculture, v.217, p.547-558, 2003.

ZOCCARATO, I. et al. Differences in performance, flesh composition and water output quality in relation to density and feeding levels in rainbow trout Oncorhynchus mykiss (Walbaum), farming. Aquaculture and Fisheries Management, v.25, p.639-647, 1994. 